\title{
APURAÇÃO DO CUSTO DA DIÁRIA DE INTERNAÇÃO HOSPITALAR: UM ESTUDO DE CASO ${ }^{1}$
}

\section{CALCULATION OF COST OF DAILY HOSPITAL HOSPITAL: A CASE STUDY}

\section{DETERMINACIÓN DEL COSTO DIARIO DE INGRESO AL HOSPITAL: UN ESTUDIO DE CASO}

\author{
PAULO CESAR SOUZA \\ Professor da UNEMAT \\ paulobbg@unemat.br
}

\author{
JOÃO HENRIQUE SCATENA \\ Instituto de Saúde Coletiva/UFMT \\ jsctena@terra.com.br
}

\begin{abstract}
RESUMO
A apuração de custos em hospitais é um processo complexo, em virtude da complexidade inerente à própria organização que tem em seu bojo uma multiplicidade de serviços e profissionais gerando simultaneamente um volume expressivo de dados que necessitam ser capturados e processados. Este artigo apresenta os resultados de uma dissertação de mestrado que ser propôs a apurar o custo da diária de internação de um hospital público. Assim, são apresentados os resultados desse estudo de caso com abordagem quantitativa, descrevendo objetivamente os passos metodológicos percorridos para a apuração do custo do paciente-dia ou diária de internação hospitalar, acompanhados dos resultados. Ficou evidente a importância do sistema de informação no processo de apuração de custos hospitalares e também a importância da informação produzida pela contabilidade na gestão dos hospitais públicos. Através da pesquisa, foi produzida uma informação de grande importância, mas que o gestor da unidade não dispunha, ou seja, o custo da diária de internação de um paciente e adicionalmente apresentou-se a comparação desse custo com a receita obtida.
\end{abstract}

PALAVRAS-CHAVE: Contabilidade de custos; Gestão de custos; Administração hospitalar.

\begin{abstract}
The calculation of costs in hospitals is a complex process, because of the complexity inherent in the organization which has within it a multitude of services and professionals while generating a significant volume of data that needs to be captured and processed. This article presents the results of a dissertation to be proposed to determine the daily cost of hospitalization in a public hospital. Thus, we present the results of this case study with a quantitative approach, describing objectively the methodological steps traversed to calculate the cost of day-patient or day hospital stay, accompanied by the results. It was evident the importance of the information system in the process of determination of hospital costs and also the importance of accounting information produced by the management of public hospitals. Through research, it was produced information of great importance, but the manager did not have the drive, the daily cost of hospitalization of a patient and additionally presented a comparison of that cost with the revenue earned.
\end{abstract}

KEYWORDS: Cost Accounting; Cost Management; Administration hospital.

\section{RESUMEN}

El cálculo de los costes en los hospitales es un proceso complejo, debido a la complejidad inherente a la organización que tiene en su interior una multitud de servicios y profesionales, mientras que la generación de un volumen importante de datos que necesita ser capturada y procesada. En este artículo se presentan los resultados de una tesis que se propone para determinar el costo diario de hospitalización en un hospital público. Por lo tanto, se presentan los resultados de este estudio de caso con un enfoque cuantitativo, que describe objetivamente los pasos metodológicos atravesadas para calcular el costo de los días-paciente o días de hospitalización, acompañado por los resultados. Era evidente la importancia del sistema de información en el proceso de determinación de los costos hospitalarios y de la importancia de la información contable producida por la gestión de los hospitales públicos. A través de la investigación, se produce información de gran importancia, pero el director no tiene la unidad, es decir, el coste diario de la hospitalización de un paciente y, además, se presenta una comparación de ese coste con los ingresos obtenidos.

PALABRAS-CLAVE: Contabilidad de Costos, Gestión de Costes, hospital de la Administración.

\footnotetext{
${ }^{1}$ Submetido em 12 de junho de 2013. Aceito em 15 de setembro de 2014. O artigo foi avaliado segundo o processo de duplo anonimato e avaliado pelos editores. Editores responsáveis: Márcio Augusto Gonçalves e Lucas Maia dos Santos. Reprodução parcial ou total e trabalhos derivativos permitidos com a citação apropriada da fonte.
} 


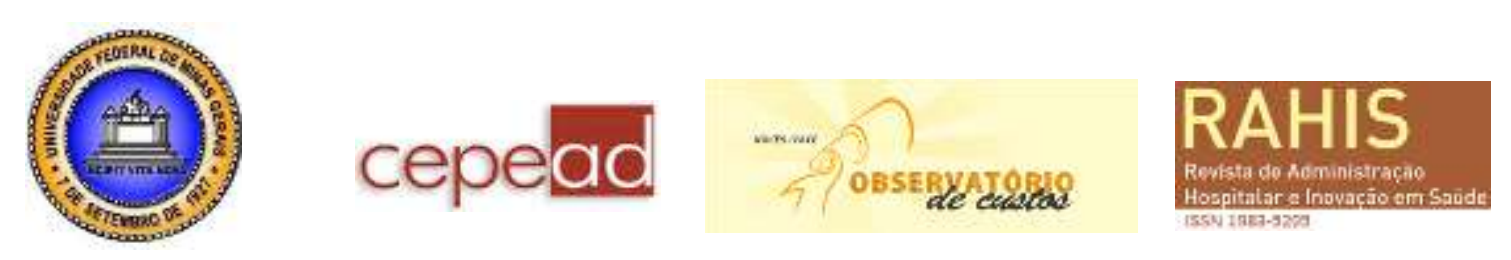

\section{INTRODUÇÃO}

Um dos grandes desafios da gestão de hospitais públicos é o aumento da eficiência. Esta eficiência é entendida como "a relação favorável entre os resultados obtidos e os recursos alocados" (MENDES, 1998, p.57). Assim, um hospital eficiente é aquele que é capaz de obter o máximo de resultados consumindo o mínimo possível de recursos. Dessa forma, a eficiência é a busca pela maximização de resultados com os mesmos recursos ou a redução do consumo de recursos mantendo-se os resultados.

Num contexto onde as necessidades são praticamente ilimitadas e os recursos são escassos, como é o caso da saúde pública, a principal preocupação deve ser no modo como estes recursos são utilizados, ou seja, a minimização dos recursos mantendo ou maximizando os resultados. Diante disso, a contabilidade de custos, mostra-se como um importante instrumento de apoio à gestão. Visando especificamente a implantação de medidas que visem aumentar a eficiência de organizações hospitalares, as informações sobre apuração e controle dos custos dessas organizações são fundamentais (BEUREN E SCHLINDWEIN, 2008). Mesmo diante da importância do sistema de apuração e gestão de custos, grande parte das organizações hospitalares não utilizam sistema de custos que seja capaz de fornecer informações úteis para a tomada de decisão ou o fazem de forma precária (ABBAS, 2001).

Assim, se o desejo é minimizar recursos e maximiar resultados o ponto de partida do processo é saber quais são os recursos empregados (custos), ou seja, quais foram os gastos com bens ou serviços utilizados na produção de outros bens ou serviços (MARTINS, 1996), em nosso caso, serviços hospitalares.

Diante da escassez de informações sobre custos no âmbito do hospital público em estudo, houve a necessidade de estruturar um sistema a fim de verificar o custo de operação do referido hospital. Diante desse desejo, a principal pergunta foi: qual objeto de custo deseja-se mensurar? O objeto de custo é definido como "qualquer coisa de que se deseja medir o custo ou que necessite de uma medida de custo separado" (CHING, 2001, p. 20). Desse modo, um objeto de custo pode ser fornecedor, linha de produtos, departamento, projeto, processo de negócio ou conjunto de atividades que realiza.

No âmbito dos hospitais, esse objeto de custos poderia ser o paciente, o procedimento, a doença, o setor, a clínica ou o hospital como um todo. Este trabalho apresenta os resultados de uma dissertação de mestrado que se propôs a apurar o custo médio da diária de internação das quatro clínicas do referido hospital. Assim, este artigo apresenta de forma objetiva os passos percorridos para a apuração desse objeto de custo escolhido, ou seja, "diária de internação hospitalar". Tecnicamente a diária de internação é chamada de paciente-dia, ou seja, é a unidade de medida que representa a assistência prestada a um paciente internado durante um dia hospitalar (MS, 2002, p. 6).

\section{ELEMENTOS FUNDENTAIS PARA A APURAÇÃO DE CUSTOS HOSPITALARES}

Um dos elementos fundamentais para a apuração de custos é a existência de um sistema de apuração e controle de custos. Segundo Tanaka et al. (2001, p.45) "para calcular os custos das atividades ou procedimentos é necessário existir um controle contábil, por meio da organização e da sistematização dos custos, com o registro dos gastos por setor ou serviço".

Para que seja possível o levantamento dos dados necessários à apuração e gestão de custos, faz-se necessário a existência na organização de um sistema de informação, baseado na sua maioria na Tecnologia da Informação (CAMPOS, 2008). A Tecnologia da Informação (TI) em Saúde corresponde a todas as formas de conhecimento relacionadas à produção, gestão e 
disseminação de informações que possuem valor de uso para solucionar ou amenizar problemas de saúde de indivíduos ou populações (VASCONCELLOS, 2002). Com a utilização de um sistema de informação baseado em recursos computacionais facilita-se a obtenção dos valores dos custos diretos, um importante elemento na formação do custo total.

De acordo com Falk (2001), existem alguns elementos que são imprescindíveis para a existência de um sistema apuração de custos. Esses componentes são: folha de pagamento, registro contábil, controle de inventário e consumo, faturamento e contas a receber, prontuário médico, controle de demanda de pacientes. Todos esses elementos geram dados para o sistema de apuração de custos hospitalares, o qual poderá gerar informações que irão orientar as decisões estratégicas.

As informações necessárias para a conformação de um sistema de apuração de custos são divididas por Beulke e Bertó (2008) em dois grupos: componentes monetários e componentes físicos, os quais são descritos a seguir.

\section{Componentes monetários}

Materiais - Nessa perspectiva, as informações sobre materiais abrangem todos os tipos de materiais que são adquiridos, estocados e consumidos pelo hospital. Esses materiais são medicamentos, materiais médico-hospitalares, materiais de limpeza, materiais de escritório, combustíveis, gases, gêneros alimentícios, peças de reposição, materiais de expediente e outros.

Em virtude do volume de informações que geralmente produzem essas movimentações, se faz necessário a existência de sistema de controle de estoque informatizado, o qual pode ser descentralizado. Dessa forma, cada setor que adquire, armazena e consome materiais tem o seu próprio sistema de controle ou esses materiais podem ser centralizados num único local, onde estaria funcionando o sistema de controle de estoques. Em geral os hospitais dividem esse trabalho com dois setores, farmácia e almoxarifado. Na farmácia há o armazenamento e controle do consumo dos medicamentos mediante as solicitações feitas pelos demais setores. No almoxarifado há o armazenamento e controle do consumo dos demais itens que não se configuram como medicamentos, registrando as saídas mediante as solicitações dos demais setores.

A informação monetária que interessa à apuração de custos no caso dos materiais é o valor dos materiais em geral consumidos no período, seja por setor, ou por pacientes específicos, dependendo de qual é o objeto de custo em foco.

Quanto à avaliação dos materiais, a mesma será realizada de acordo com o objetivo do controle de materiais, se para fins fiscais ou gerenciais. Se para fins fiscais o ideal seria pelo método estabelecido pela legislação do imposto de renda chamado custo médio, pelo qual o valor de cada produto será o valor médio de cada produto em estoque, especificado pelo tipo de produto. Se para fins gerenciais, o ideal é utilizar-se do valor atual dos produtos, ou seja, se não for possível o preço de mercado, o último valor de aquisição.

Pessoal - As informações sobre gastos com pessoal que interessa ao sistema de custos são fornecidas pelo sistema de gerenciamento de pessoal. Tais informações necessitam ser encaminhadas no formato necessário dependendo do sistema de custeio adotado. Em geral esses custos são agrupados por centro de custos. Assim, a informação monetária que a apuração de custos necessita geralmente se refere aos valores gastos com pessoal por centro de custos. Os valores considerados serão todos os gastos com pessoal, ou seja, salários, encargos, férias, adicional de férias, décimo terceiro e outros.

Depreciação - Os custos com depreciação são fornecidos pelo sistema de controle patrimonial, o qual deverá controlar o registro e avaliação de todos os bens que compõem o patrimônio da instituição. Com base nos valores dos bens, geralmente registrados por centro de 
custos calcula-se a taxa de depreciação, a qual representa o desgaste do bem no período que foi utilizado nas atividades da organização. Em hospitais públicos, muitas vezes no momento do cálculo do custo esse elemento é desconsiderado.

Custos Gerais - No item custos gerais, são agrupados os itens de custo que não estão incluídos nos demais grupos acima, como energia, água, serviços de terceiros, etc. Esses custos terão seus valores obtidos junto á contabilidade e quando for possível identificar com exatidão para qual centro de custos foi alocado o referido gasto ele poderá ser apropriado diretamente. Caso isso não seja possível, será necessária a utilização de algum critério de rateio. No caso do consumo de água, por exemplo, a forma mais simples de distribuição do gasto por rateio seria de acordo com a representatividade dos metros quadrados ocupados por cada setor.

Custos de comercialização - Os custos de comercialização são custos que ocorrem geralmente em hospitais privados, tendo como principais fontes a legislação e os contratos de serviços.

É importante lembrar que a classificação proposta pelos autores Beulke e Bertó (2008) é característica de instituições hospitalares privadas. No entanto, ao aplicar essa classificação às instituições hospitalares públicas pode-se suprimir o último item, restando então como principais componentes dos custos hospitalares os materiais, pessoal, depreciação e custos gerais.

Convém ainda destacar que o montante dos três primeiros componentes monetários na classificação proposta por Beulke e Bertó (2008), ou seja, materiais, pessoal e depreciação, representam os tipos de custos diretos mais comuns da atividade hospitalar.

Nesse processo de classificação dos custos em grupos, o Ministério da Saúde (MS, 2006, p. 31) propõe os seguintes grupos: pessoal, serviços de terceiros, materiais de consumo, despesas gerais e depreciação.

Alguma variação em geral pode acontecer quanto à forma de classificação, no entanto, os principais elementos são sempre os mesmos.

\section{Componentes físicos}

Os componentes físicos que estão presentes nas organizações hospitalares demonstram inúmeras circunstâncias e podem ser agrupados segundo Beulke e Bertó (2008) nos seguintes itens:

- Quantidade de materiais movimentados pelo sistema de materiais;

- Montante de tempo de trabalho por procedimento como horas de cirurgia, número de pacientes-dia, tempo de duração de um exame, etc.

- Produção mensal: número de internações, número de exames realizados, número de atendimentos, $\mathrm{kg}$ de roupa lavada, peça esterilizada, etc.

Resta verificar como todos esses componentes monetários e físicos são processados para que finalmente seja possível o conhecimento dos custos dos serviços.

\section{METODOLOGIA}

Este artigo apresenta os resultados de uma pesquisa realizada no Hospital Municipal "Roosevelth Figueiredo Lira", em Barra do Bugres, cidade localizada a 155 km de Cuiabá, capital de Mato Grosso. O Hospital Municipal de Barra do Bugres é uma instituição pública, mantida com recursos municipais, convênio com a SES/MT e vinculado ao Sistema Único de Saúde. De acordo com Cadastro Nacional de Estabelecimentos de Saúde - CNES (CNES, 2007), o hospital tem sua estrutura composta por 80 leitos, sendo estes divididos entre as clínicas médica, cirúrgica, obstétrica e pediátrica. 


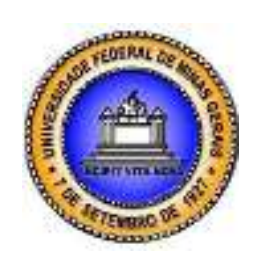

Utilizando esse tipo de classificação, o objetivo é que após o levantamento do custo total por cada centro de custos, tais custos sejam rateados entre eles, de acordo com a metodologia a ser utilizada de modo que os custos dos centros administrativos e intermediários sejam transferidos para os finais e externos.

\section{Quadro 1 - Classificação dos centros de custos por tipo, H. M. Barra do Bugres, 15/10 a 14/11/2008.}

\begin{tabular}{|l|l|l|l|}
\hline \multicolumn{1}{|c|}{ Administrativo } & \multicolumn{1}{c|}{ Intermediário } & \multicolumn{1}{c|}{ Final } & \multicolumn{1}{c|}{ Externo } \\
\hline Administração & Centro cirúrgico & Clínica Cirúrgica & Unidades Externas \\
\hline Farmácia & $\begin{array}{l}\text { Centro de Mat. } \\
\text { Esterilizados }\end{array}$ & $\begin{array}{l}\text { Clínica Ginecológica } \\
\text { e Obstétrica }\end{array}$ & \\
\hline Faturamento/estatística & Estabilização & Clínica Médica & \\
\hline Gasoterapia & Laboratório & Clínica Pediátrica & \\
\hline Gerência de Enfermagem & Lavanderia & $\begin{array}{l}\text { Pronto Socorro } \\
\text { /Ambulatório }\end{array}$ & \\
\hline Manutenção & Limpeza & & \\
\hline Recepção & Nutrição & & \\
\hline Telefonia & Radiologia & & \\
\hline Transporte & Ultra-sonografia & & \\
\hline
\end{tabular}

\section{Segundo passo}

Apuração dos custos diretos: Conforme já destacado, as informações básicas para o processo de apuração de custos podem ser divididas em componentes monetários e componentes físicos. Os componentes monetários se dividem nos seguintes grupos de informações: materiais, pessoal e custos gerais. Estes devem ser expressos em moeda corrente.

Os custos diretos existentes na instituição eram os seguintes: Materiais, pessoal e encargos e serviço de terceiros, os quais são expressos em moeda corrente e fazem parte dos componentes monetários apresentados. Assim, esses três elementos que compõem os custos diretos serão apresentados a seguir.

Materiais diretos: No que tange ao controle de materiais, o ideal é que ele seja realizado de forma centralizada. No caso de um hospital, esse controle deveria ser realizado pelo almoxarifado central e pela farmácia. No entanto, no hospital em análise isso não foi possível, em virtude da inexistência de almoxarifado. O software desenvolvido foi utilizado para controlar o material consumido em todo o hospital, especificando em cada saída de material o setor ou centro de custo de destino. Esse controle possibilitou a emissão de relatórios demonstrando o valor dos materiais consumidos no período por cada um dos centros de custos.

As informações sobre pessoal não foram levantadas através do sistema de informação desenvolvido para o hospital, em virtude do pouco tempo disponível para o seu desenvolvimento. Através do acesso à relação de funcionários com seus respectivos salários e encargos, foi possível apurar o custo com pessoal por centro de custos.

Os custos diretos com os serviços de terceiros tiveram seus valores obtidos junto à administração do hospital e junto à contabilidade da prefeitura municipal.

Pessoal e encargos: $O$ custo com pessoal e encargos é um elemento de grande importância na composição dos custos totais do hospital. Eles foram obtidos junto ao departamento de pessoal da prefeitura e organizados em planilha de modo a permitir o cálculo do custo com pessoal e encargos por centro de custos. É importante destacar que a classificação de custos com pessoal e encargos foi utilizada neste trabalho se referindo ao custo com os servidores com vínculo com o hospital, seja concursado, contratado ou nomeado. Existem ainda outros custos que são classificados contabilmente como serviço de terceiros, como por exemplo, 
a remuneração dos médicos plantonistas do pronto-socorro, os quais na verdade também representam custos com pessoal, no entanto, para os fins deste trabalho foi mantida a classificação contábil.

Assim, o Quadro 2 apresenta o resumo dos valores apurados com materiais de consumo para o hospital no período analisado.

Quadro 2 - Distribuição dos custos (em R\$) com materiais de consumo, segundo centro de custos e tipo de material, H. M. Barra do Bugres, 15/10 a 14/11/2008.

\begin{tabular}{|c|c|c|c|c|c|c|c|c|c|c|c|c|c|c|}
\hline Cent & $\begin{array}{c}\text { Med. E } \\
\text { maberiaks } \\
\text { hup. }\end{array}$ & $s$ & $\begin{array}{l}\text { Materinis } \\
\text { de Linrerat }\end{array}$ & $*$ & 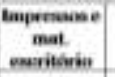 & $*$ & $\begin{array}{l}\text { Choeros } \\
\text { alianest, II } \\
\text { denewt }\end{array}$ & $*$ & $\begin{array}{l}\text { Camb e } \\
\text { asiophio }\end{array}$ & *6 & $\begin{array}{l}\text { Mat. } \\
\text { Mamat. }\end{array}$ & 96 & Totel & s. \\
\hline Adme & 0,00 & 0,00 & 103,70 & 14,74 & 447,61 & 63,99 & 121.71 & 17,20 & 0.00 & 0,00 & 30,50 & $4,3 x$ & 703,91 & 11200 \\
\hline Vermicia & 0,00 & 0,00 & 0.00 & 0,00 & 4,14 & 100 & 0.00 & 0,00 & 0.00 & 0,00 & 0.00 & 0.00 & & 400 \\
\hline Vaturumento/esatatistica & 0,00 & 0,00 & 0,00 & 0,00 & 353,60 & 100 & 0.00 & 0,00 & 0,00 & 0,00 & 0,00 & 0,00 & 353,60 & 00100 \\
\hline Slasoteripia & 0,00 & 0,00 & 0,00 & 0,00 & $0, \infty$ & 0,00 & 0.00 & 0,00 & $6.116,50$ & 100 & 0,00 & 0,001 & $6.116,90$ & 0900 \\
\hline Ger. de Fieficmangan & 0,00 & 0,00 & 0,00 & 0,00 & 1,24 & 100 & 0,00 & 0,00 & 0,00 & 0,00 & 0,00 & 0,00 & & 4300 \\
\hline Maanutençalo & 0,00 & 0,00 & 0,00 & 0,00 & 0,00 & 0,00 & 0,00 & 0,00 & 0.00 & 0,00 & 0.00 & 0,00 & 0,00 & 00 \\
\hline Recopclio & 2,30 & 5,90 & 0,00 & 0,00 & 1,73 & 4,31 & 0.00 & 0,00 & 0.00 & 0,00 & 36,00 & 89,73 & 40,12 & 2300 \\
\hline Febfonia & 0.00 & 0,00 & 0.00 & 0,00 & 41,22 & 100 & 0,00 & 0.00 & 0.00 & 0,00 & 0.00 & 0,00 & 41,22 & 2300 \\
\hline Tranepork & 0,00 & 0,00 & 0,00 & 0,00 & 0,00 & 0,00 & 0,00 & 0,00 & $2.316,42$ & 100 & 0,00 & 0,00 & $2.316,42$ & 2300 \\
\hline Centro ciriurgica & $3.043,42$ & 86,30 & 290,50 & 7,37 & 2200.04 & 6,25 & 0.00 & 0,00 & 0,00 & 0,00 & 0,00 & 0,00 & $3.522,96$ & 66900 \\
\hline CMII & 370.08 & 43,53 & 389,47 & $45, \mathrm{H2}$ & 90,37 & 10,5 & 0,00 & 0.00 & 0.00 & 0,00 & 0.90 & 0,00 & 1550,00 & 90100 \\
\hline Hatabiliasçla & $2.040,97$ & 99,00 & 6.61 & 0,22 & 21,73 & $0,7 z$ & 0,00 & 0,00 & 0,00 & 0,00 & 0,00 & 0,00 & $3.009,31$ & 1100 \\
\hline Laboratóno & 379.70 & 59,00 & 162.44 & 25,52 & 94,31 & 14,10 & 0,00 & 0.90 & 0,00 & 0,00 & 0.00 & 0,00 & $636,5 \mathrm{H}$ & 18900 \\
\hline Lavasikaria & 29,45 & 2,01 & $1.432,16$ & V7, $\mathrm{s}$ & 4.00 & 0,34 & 0,00 & 0,00 & 0.00 & 0,00 & 0.00 & 0,00 & $1.466,57$ & 7300 \\
\hline K.iangesa & 121,10 & 99,76 & 0,00 & 0,00 & 0,24 & 0,24 & 0,00 & 0,00 & 0,00 & 0,00 & 0,00 & 0,00 & 121,30 & 9100 \\
\hline Nutriçlo & sos,z1 & 5,70 & 205,85 & 1,51 & 44,34 & 0,25 & $14.469,25$ & 92,34 & 0,00 & 0,00 & 26,20 & 0,17 & $15.668,92$ & 2300 \\
\hline Radiologia & $2.531,54$ & 99,41 & 0.00 & 0,00 & 14,52 & 0.97 & 0,00 & 0.00 & 0.00 & 0,00 & 0.00 & 0,00 & 2146,66 & 6000 \\
\hline Bitri-noeogentia & no,40 & 46,80 & 0.00 & 0,00 & 91,31 & 53.20 & 0,00 & 0,00 & 0,00 & 0,00 & 0.00 & 0,00 & 171,71 & 11100 \\
\hline Clinica Cirürgica & $5.126,62$ & 92,22 & 400,20 & 7,36 & 22,06 & 0,41 & 0,00 & 0.00 & 0.00 & 0,00 & 0.00 & 0,00 & $5.558, k$ ? & 7700 \\
\hline Clínica 0.0 . & 4001,34 & 92,58 & 156,54 & 2,90 & 182,95 & 3,39 & 0.00 & 0.00 & 0.00 & 0,00 & 60,50 & 1,12 & $3.391,42$ & 2300 \\
\hline Clinica Mbrtica & $6.201,90$ & 91,10 & 463.22 & $6,11$. & 136,29 & 2,00 & 0,00 & 0.00 & 0.00 & 0,00 & 0.00 & 0,00 & $6.503,41$ & 1100 \\
\hline Clinica Motiátric & $3.111,21$ & 15,3* & 629,701 & 10,32 & 190 , & 3,11 & 0.00 & 0.00 & 0,00 & 0,00 & 35,00 & 0.92 & $9.986,66$ & 6100 \\
\hline p.s./Aunbulatirio & $11.176,90$ & 93,02 & 627,20 & 5,22 & 211.97 & 1,76 & 0.00 & 0,00 & 0,00 & 0,00 & 0.00 & 0,00 & $12.014,77$ & moo \\
\hline Deidadea & 70.74 & 100 & 0.00 & 0,00 & 0,00 & 0,00 & 0,00 & 0,00 & 0,00 & 0,00 & 0.00 & 0,00 & 70,74 & 1400 \\
\hline & $43.415,01$ & 58,91] & 4.875.94 & 6,62 & $2.179,94$ & 2,95 & 990,96 & 19,80 & 8. 432,97 & 11.44 & 208,70 & $0,2 \mathrm{~K}$ & 7969,27 & 7300 \\
\hline
\end{tabular}

Desse modo, a Tabela 1 apresenta o valor do custo com pessoal e encargos levantados no período em análise para cada centro de custo do hospital.

Tabela 1- Distribuição dos custos com pessoal e encargos, segundo centro de custo, H. M. Barra do Bugres, 15/10 a 14/11/2008.

\begin{tabular}{l|rc}
\hline Centro de custo & Valor & $\%$ \\
\hline Administração & $3.891,79$ & 1,45 \\
Farmácia & $10.108,24$ & 3,78 \\
Faturamento/estatística & $4.714,64$ & 1,76 \\
Gasoterapia & 0,00 & 0,00 \\
Gerência de Enfermagem & $22.251,15$ & 8,31 \\
Manutenção & $2.495,05$ & 0,93 \\
Recepção & $14.803,27$ & 5,53 \\
Telefonia & 614,10 & 0,23 \\
Transporte & $5.208,86$ & 1,95 \\
Centro cirúrgico & $12.123,96$ & 4,53 \\
Centro de Mat. Esterilizados & $9.519,37$ & 3,56 \\
Estabilização & 0,00 & 0,00 \\
Laboratório & $13.076,92$ & 4,88 \\
Lavanderia & $4.261,55$ & 1,59 \\
Limpeza & $11.895,09$ & 4,44 \\
Nutrição & $14.337,75$ & 5,36 \\
Radiologia & $4.123,64$ & 1,54 \\
Ultra-sonografia & $4.564,59$ & 1,70 \\
\hline
\end{tabular}




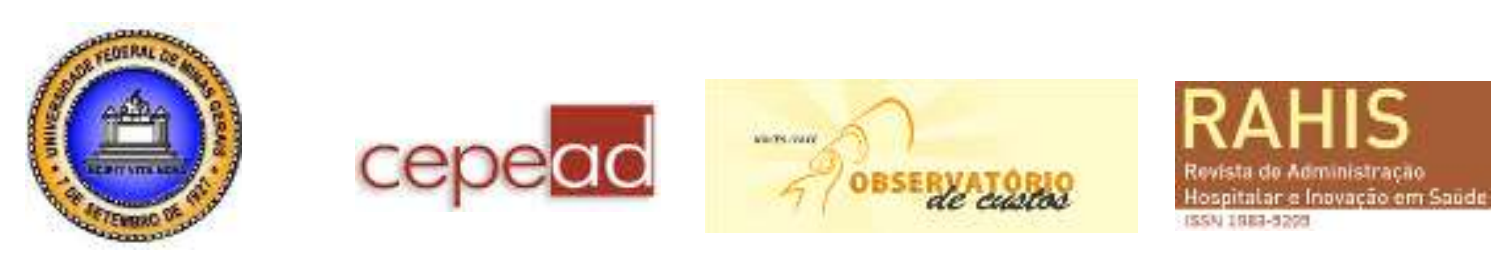

\begin{tabular}{l|rr}
\hline Clínica Cirúrgica & $17.431,45$ & 6,51 \\
Clínica Ginecológica e Obstétrica & $33.946,15$ & 12,68 \\
Clínica Médica & $26.828,17$ & 10,02 \\
Clínica Pediátrica & $22.460,65$ & 8,39 \\
P.S./Ambulatório & $29.079,25$ & 10,86 \\
\hline Total & $\mathbf{2 6 7 . 7 3 5 , 6 5}$ & $\mathbf{1 0 0 , 0 0}$ \\
\hline
\end{tabular}

Serviço de terceiros: Nesse item serviço de terceiros estão inclusos os serviços prestados ao hospital por pessoas físicas e também pessoas jurídicas, não havendo a distinção segundo a classificação jurídica.

Assim, a Tabela 2 apresenta a distribuição dos valores de serviço de terceiros por centro de custo do hospital no período em análise.

Tabela 2 - Distribuição dos custos com serviço de terceiros, segundo centros de custos, H. M. Barra do Bugres, 15/10 a 14/11/2008.

\begin{tabular}{l|rr}
\hline Centro de custo & \multicolumn{1}{|c}{ Valor } & \multicolumn{1}{c}{ \% } \\
\hline Administração & $1.173,62$ & 1,27 \\
Farmácia & 16,00 & 0,02 \\
Faturamento/estatística & $1.914,45$ & 2,08 \\
Recepção & $3.567,22$ & 3,87 \\
Telefonia & $3.657,34$ & 3,97 \\
Transporte & $1.125,00$ & 1,22 \\
Centro cirúrgico & $6.500,00$ & 7,05 \\
Laboratório & $22.281,05$ & 24,17 \\
Limpeza & $1.341,17$ & 1,46 \\
Nutrição & $4.671,04$ & 5,07 \\
Clínica cirúrgica & 350,00 & 0,38 \\
Clínica G. O. & $1.640,09$ & 1,78 \\
Clínica Médica & $3.750,00$ & 4,07 \\
Clínica Pediátrica & 50,00 & 0,05 \\
P.S./Ambulatório & $40.130,00$ & 43,54 \\
\hline Total & $\mathbf{9 2 . 1 6 6 , 9 8}$ & $\mathbf{1 0 0}$ \\
\hline
\end{tabular}

\section{Terceiro Passo}

Apuração dos custos indiretos gerais: Os principais custos gerais, ou custos indiretos gerais, geralmente presentes nas organizações hospitalares são: telefonia, água e energia.

Em virtude do setor de telefonia ser classificado como um centro de custo e do hospital não pagar pela água utilizada por possuir poço artesiano e receber ainda água do serviço municipal de distribuição de água, foi considerado como custo indireto geral apenas o custo com energia, o qual totalizou R $\$ 11.924,57$ no período. Este custo foi rateado aos diversos centros de custos através da aplicação da metodologia apresentada por Saraiva (2006). O ponto inicial da metodologia é o levantamento dos pontos de luz e equipamentos existes nos centros de custos, bem como a carga horária de trabalho de cada um deles, e com base nisso o estabelecimento dos seguintes pesos:

Peso 1: utilizado para identificar cada ponto de luz existente por centro de custo;

Peso 2: identifica os equipamentos que demandam um consumo de energia não superior a doze horas, como os computadores, impressoras, televisores, máquina de xérox, equipamento de som, balanças digitais, exaustores, ar-condicionado, etc.

Peso 3: considerando-se que alguns setores do hospital funcionam 24 horas, esse peso é aplicado aos equipamentos semelhantes aos do peso 2 , mas que funcionam de forma ininterrupta;

Peso 4: aplicado aos equipamentos de uso diário, que demandam um consumo maior de energia: máquinas de lavar, secadoras e geladeiras industriais, autoclaves, esterilizadoras, etc. 


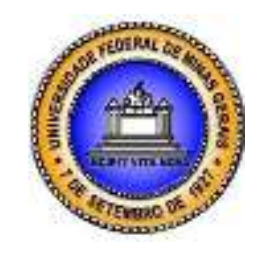

\section{Quarto Passo}

Apuração dos custos totais: Após o levantamento dos itens anteriores e antes de realizar o rateio entre os vários centros de custos, a Tabela 4 apresenta de forma consolidada os custos totais por cada centro de custo e também o valor do custo indireto geral, o qual também será rateado a seguir aos centros de custos.

Tabela 4 - Distribuição dos custos totais, segundo centro de custos, H. M. Barra do Bugres, 15/10 a 14/11/2008.

\begin{tabular}{|c|c|c|c|c|c|c|c|c|}
\hline \multirow[b]{2}{*}{ Centro de custo } & \multicolumn{2}{|c|}{$\begin{array}{l}\text { Mat. De } \\
\text { Consumo }\end{array}$} & \multicolumn{2}{|c|}{$\begin{array}{l}\text { Pessoal e } \\
\text { Encargos }\end{array}$} & \multicolumn{2}{|c|}{$\begin{array}{l}\text { Serv. de } \\
\text { Terceiros }\end{array}$} & \multicolumn{2}{|c|}{ Total } \\
\hline & $\mathbf{R} \$$ & $\% *$ & $\mathbf{R} \$$ & $\% *$ & $\mathbf{R} \$$ & $\% *$ & $\mathbf{R} \$$ & $\%$ \\
\hline Administração & 703,91 & 12,2 & $3.891,79$ & 67,5 & $1.173,62$ & 20,3 & $5.769,32$ & $\overline{1,3}$ \\
\hline Farmácia & 4,14 & 0,0 & $10.108,24$ & 99,8 & 16,00 & 0,2 & $10.128,38$ & 2,3 \\
\hline Faturamento/est. & 353,60 & 5,1 & $4.714,64$ & 67,5 & $1.914,45$ & 27,4 & $6.982,69$ & 1,6 \\
\hline Gasoterapia & $6.116,50$ & 100 & 0,00 & 0,0 & 0,00 & 0,0 & $6.116,50$ & 1,4 \\
\hline Gerência de Enf. & 1,24 & 0,0 & $22.251,15$ & 100 & 0,00 & 0,0 & $22.252,39$ & 4,9 \\
\hline Manutenção & 0,00 & 0,0 & $2.495,05$ & 100 & 0,00 & 0,0 & $2.495,05$ & 0,6 \\
\hline Recepção & 40,12 & 0,2 & $14.803,27$ & 80,4 & $3.567,22$ & 19,4 & $18.410,61$ & 4,1 \\
\hline Telefonia & 41,22 & 1,0 & 614,10 & 14,2 & $3.657,34$ & 84,8 & $4.312,66$ & 1,0 \\
\hline Transporte & $2.316,42$ & 26,8 & $5.208,86$ & 60,2 & $1.125,00$ & 13,0 & $8.650,28$ & 1,9 \\
\hline Centro cirúrgico & $3.522,96$ & 15,9 & $12.123,96$ & 54,7 & $6.500,00$ & 29,4 & $22.146,92$ & 5.0 \\
\hline Centro de Mat. Est. & 850,09 & 8,2 & $9.519,37$ & 91,8 & 0,00 & 0,00 & $10.369,46$ & 2,3 \\
\hline Estabilização & $3.009,31$ & 100 & 0,00 & 0,0 & 0,00 & 0,0 & $3.009,31$ & 0,7 \\
\hline Laboratório & 636,58 & 1,8 & $13.076,92$ & 36,3 & $22.281,05$ & 61,9 & $35.994,55$ & 8,1 \\
\hline Lavanderia & $1.466,57$ & 25,6 & $4.261,55$ & 74,4 & 0,00 & 0,0 & $5.728,12$ & 1,3 \\
\hline Limpeza & 121,39 & 0,9 & $11.895,09$ & 89,1 & $1.341,17$ & 10,0 & $13.357,65$ & 3,0 \\
\hline Nutrição & $15.668,92$ & 45,2 & $14.337,75$ & 41,4 & $4.671,04$ & 13,4 & $34.677,71$ & 7,8 \\
\hline Radiologia & $2.846,66$ & 40,8 & $4.123,64$ & 59,2 & 0,00 & 0,0 & $6.970,30$ & 1,6 \\
\hline Ultra-sonografia & 171,78 & 3,6 & $4.564,59$ & 96,4 & 0,00 & 0,0 & $4.736,37$ & 1,1 \\
\hline Clínica Cirúrgica & $5.558,87$ & 23,8 & $17.431,45$ & 74,7 & 350,00 & 1,5 & $23.340,32$ & 5,2 \\
\hline Clínica G.O. & $5.391,42$ & 13,2 & $33.946,15$ & 82,8 & $1.640,09$ & 4,0 & $40.977,66$ & 9,2 \\
\hline Clínica Médica & $6.805,41$ & 18,2 & $26.828,17$ & 71,8 & $3.750,00$ & 10,0 & 37.383,57 & 8,4 \\
\hline Clínica Pediátrica & $5.986,66$ & 21,0 & $22.460,65$ & 78,8 & 50,00 & 0,2 & 28.497,31 & 6,4 \\
\hline P.S./Ambulatório & $12.014,77$ & 14,8 & $29.079,25$ & 35,8 & $40.130,00$ & 49,4 & $81.224,02$ & 18,2 \\
\hline Unidades externas & 70,74 & 100 & 0,00 & 0,00 & 0,00 & 0,0 & $\mathbf{7 0 , 7 4}$ & $\mathbf{0 , 0}$ \\
\hline Sub-Total & $73.699,27$ & 17,0 & $267.735,65$ & 61,8 & $92.166,98$ & 21,2 & 433.601,90 & 97,3 \\
\hline Custos ind. Gerais & & & & & & & $11.924,57$ & 2,7 \\
\hline Custos totais & 73.699,27 & 16,5 & $267.735,65$ & 60,1 & 92.166,98 & 20,7 & 445.526,47 & 100 \\
\hline
\end{tabular}

* Percentual em relação ao total do centro de custo específico.

\section{Quinto Passo}

Rateio dos custos entre os centros de custos: Após a distribuição do custo com a energia entre os centros de custos utilizando a metodologia de rateio especificada acima, obtémse o custo total por cada centro de custos. A partir de então será realizado o rateio dos custos dos centros administrativos e intermediários para os centros finais e externos, embora os externos não sejam nosso foco de análise.

Para realizar isso, duas coisas são importantes: os componentes físicos (BEULKE e BERTÓ, 2008), que serão utilizados como critério de rateio (Quadro 3) e a definição da metodologia de alocação dos custos.

Para a realização do rateio dos custos dos centros administrativos e intermediários aos finais (produtivos) e externos foi necessário definir qual metodologia de alocação seria utilizada. De acordo com Falk (2001) e MS (2006) existem três tipos de metodologia: alocação 
direta, alocação seqüencial ou escalonar e alocação recíproca ou matricial. Neste trabalho foi utilizada a metodologia de alocação recíproca ou matricial, por se mostrar a mais adequada para o que se propunha.

\section{Quadro 3 - Componentes físicos utilizados com critério de rateio, H. M. Barra do Bugres, $15 / 10$ a $14 / 11 / 2008$.}

\begin{tabular}{|c|c|c|}
\hline $\begin{array}{c}\text { Tipo de Centro } \\
\text { de Custos }\end{array}$ & Centro de Custos & Critério de rateio \\
\hline Administrativo & Administração & Servidores por centro de custos \\
\hline Administrativo & Farmácia & $\mathrm{N}^{\mathrm{o}}$. de itens encaminhados aos centros de custos \\
\hline Administrativo & Faturamento/estatística & Internação faturada por centro de custos \\
\hline Administrativo & Gasoterapia & Pontos com fluxômetro ponderados pela utilização \\
\hline Administrativo & Ger. de Enfermagem & Servidores por centro de custos subordinados \\
\hline Administrativo & Manutenção & Metros quadrados por centro de custos \\
\hline Administrativo & Recepção & Número de internações processadas por clínica \\
\hline Administrativo & Telefonia & Ramais por centro de custos \\
\hline Administrativo & Transporte & Km rodados para cada centro de custos \\
\hline Intermediário & Centro cirúrgico & Horas de cirurgia ponderadas por tipo de proced. \\
\hline Intermediário & $\mathrm{CME}$ & $\mathrm{N}^{\circ}$. de pacotes e caixas por centro de custos \\
\hline Intermediário & Estabilização & $\mathrm{N}^{\circ}$. de pacientes ponderados pela permanência \\
\hline Intermediário & Laboratório & $\mathrm{N}^{\circ}$. de exames solicitados por centro de custos \\
\hline Intermediário & Lavanderia & Kg de roupa lavada destinada ao centro de custos \\
\hline Intermediário & Limpeza & $\mathrm{M}^{2}$ por centro ponderados pelo grau de severidade \\
\hline Intermediário & Nutrição & $\mathrm{N}^{\circ}$. de refeições ponderadas para cada centro \\
\hline Intermediário & Radiologia & $\mathrm{N}^{\mathrm{o}}$. de exames solicitados por centro de custos \\
\hline Intermediário & Ultra-sonografia & $\mathrm{N}^{\circ}$. de exames solicitados por centro de custos \\
\hline Final & Clínica Cirúrgica & Nenhum \\
\hline Final & Clínica G. O. & Nenhum \\
\hline Final & Clínica Médica & Nenhum \\
\hline Final & Clínica Pediátrica & Nenhum \\
\hline Final & P.S./Ambulatório & Nenhum \\
\hline Externo & Unidades externas & Nenhum \\
\hline
\end{tabular}

Essa metodologia, reconhece a reciprocidade entre os centros de custos, ou seja, ela reconhece que os centros de custos administrativos e intermediários não apenas transferem seus custos até que eles cheguem aos centros finais ou produtivos, mas que um centro administrativo ou intermediário pode tanto prestar como receber serviços. Essa metodologia é complexa já que se utiliza de álgebra matricial para a realização dos cálculos, em virtude disso, foi desenvolvido no software implantado junto ao hospital um módulo para apuração de custos que calcula essa distribuição recíproca dos custos de forma facilitada. O processo realizado pelo sistema é demonstrado na Figura 1. 


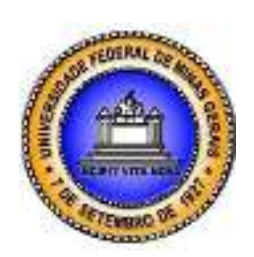

sobre seus custos. Os dados mostram o centro de custo que apresentou o maior custo da diária foi a clínica Ginecológica e Obstétrica e na comparação com as receitas médias, verifica-se que o menor déficit foi registrado pela Clínica Pediátrica.

\section{REFERÊNCIAS}

ABBAS, K. Gestão de custos hospitalares. Dissertação de mestrado em engenharia de produção. Santa Catarina: UFSC, 2006.

BEULKE, R; BERTÓ, J.D. Gestão de custos e resultados na saúde: hospitais, clínicas, laboratórios e congêneres. 4. ed. rev. atualizada e ampliada. São Paulo: Saraiva, 2008.

BEUREN, I. M.; SCHLINDWEIN, N. F. Uso do Custeio por Absorção e do Sistema RKW para gerar informações gerenciais: Um estudo de caso em hospital. Associação Brasileira de Custos, Vol. III, nº. 2, 2008. Disponível em: www.abcustos.org.br.

CAMPOS, M. Apostila de sistema de informação. Disponível em: http://www.marcelocampos.com.br/academico/tur/download/aula007sistema.pdf (acesso dia 16.09.2008).

CHING, Y.H. Manual de custos de instituições de saúde: sistemas tradicionais de custos e sistema de custeio baseado em atividades (ABC). São Paulo: Atlas, 2001.

DA SAÚDE. Secretaria de Atenção à Saúde. DATASUS. Cadastro Nacional de Estabelecimentos de Saúde - CNES. Brasília; 2007. [acesso dia 27 de novembro de 2007]. Disponível em: http://cnes.datasus.gov.br/Lista_Es_Nome.asp?VTipo=0

FALK, J.A. Gestão de custos para hospitais: conceitos, metodologias e aplicações. São Paulo: Atlas, 2001.

FALK, J.A. Tecnologia de informação para gestão de custos e resultados no hospital: considerações e dicas para implantação de um modelo brasileiro. São Paulo: Atlas, 2008.

MARTINS, E. Contabilidade de custos. 5ª ed. rev. São Paulo: Atlas, 1996.

MARTINS, G.A. Métodos convencionais e não-convencionais e a pesquisa em administração. Caderno de pesquisas em administração. V. 00, N. 0, 2. Semestre, 2004.

MENDES, EV. A organização da saúde no nível local. São Paulo: Hucitec, 1998.

MINISTÉRIO DA SAÚDE. Gabinete Ministerial. Portaria 312 de 02 de maio de 2002. Dispõe sobre a Padronização da Nomenclatura do Censo Hospitalar. Brasília, DF; 2002.

MINISTÉRIO DA SAÚDE. Secretaria de Ciência, Tecnologia e Insumos Estratégicos. Departamento de Economia da Saúde. Programa Nacional de Gestão de Custos. Manual Técnico de Custos: conceitos e metodologia. Brasília, DF; 2006.

SARAIVA, M.C. Investigação da mensuração de custos na formação dos preços dos serviços hospitalares: um foco na diária hospitalar [dissertação de mestrado]. Natal: Programa 
Multiinstitucional e Inter-regional de Pós-Graduação em Ciências Contábeis - UNB, UFPB, UFPE, UFRN, 2006.

TANAKA, Y.O., MELO, C. Avaliação de Programas de Saúde do Adolescente: um modo de fazer. Editora da Universidade de São Paulo. São Paulo: 2001.

VASCONCELlOS, M.M; MORAES, I. H.; CAVALCANTE, M.T.L. Política de saúde e potencialidades de uso das tecnologias de informação. Saúde em Debate 2002; 26 Supl. 61: 219-235. 